\title{
Golpe de calor y sus complicaciones: a propósito de un caso en el servicio de urgencias
}

\author{
Heat stroke and its complications: a case in emergency department \\ Rafael A. Reyes-Monge* y Tonantzin S. Ibarra-Ocegueda \\ Servicio de Urgencias, Hospital General del Estado de Sonora, Hermosillo, Sonora, México
}

\section{Introducción}

El golpe de calor es la condición más extrema en el espectro de las enfermedades atribuidas al calor. Se caracteriza por tener el antecedente de exposición prolongada a altas temperaturas que condicionan un incremento de la temperatura corporal central. Clínicamente se observan disfunción del sistema nervioso central, falla multiorgánica e hipertermia $\left(>40^{\circ} \mathrm{C}\right)^{1}$.

En México, las alteraciones relacionadas con la exposición a alzas térmicas son registradas de manera usual en primavera y verano, con un promedio de 1200 casos al año y una mortalidad de 15 casos $^{2}$.

La incidencia verdadera del golpe de calor es incierta, ya que con frecuencia no es diagnosticado de manera adecuada, pues no es una patología común en algunos Estados del país, y como consecuencia la morbimortalidad debe ser mayor.

\section{Presentación del caso}

Varón de 28 años, originario del Estado de México, jornalero de oficio, con antecedente de acudir al estado de Sonora 3 días previos a su ingreso al servicio (sin aclimatación adecuada). Se desconocen antecedentes heredofamiliares, personales no patológicos y personales patológicos. Referido de una clínica rural al iniciar su padecimiento actual, el día de su ingreso, tras encontrarse trabajando en el campo expuesto a altas temperaturas (documentadas de $43^{\circ} \mathrm{C}$ ) presenta deterioro neurológico caracterizado por pérdida del estado de despierto, además de crisis convulsivas tónico-clónicas con supraversión de la mirada y pérdida de control de los esfínteres. A su llegada al servicio de urgencias se detectan los siguientes signos vitales: presión arterial 120/70 $\mathrm{mmHg}$, frecuencia cardiaca 120 latidos por minuto, frecuencia respiratoria 40 respiraciones por minuto, temperatura corporal central 42 ${ }^{\circ} \mathrm{C}$, escala de coma de Glasgow 7 puntos (O2, V2, M3), pupilas isocóricas, palidez tegumentaria, mucosa oral deshidratada, tórax con uso de musculatura accesoria, precordio con tendencia a la taquicardia, extremidades flexionadas, con respuesta plantar equívoca. Ante el deterioro neurológico se decide asegurar la vía aérea, además de realizar una tomografía computada simple de cráneo, en la cual no se aprecian alteraciones evidentes. Se le realizan paraclínicos, entre los que destacan trombocitopenia de 57,000 y gasométricamente una acidosis metabólica grave de anión gap normal. Presenta lesión renal AKIN 2, hiperpotasemia moderada con fibrinógeno disminuido y dímero $D$ incrementado, por lo que se integró el diagnóstico de coagulación intravascular diseminada (Tabla 1). Se inició tratamiento disminuyendo la temperatura corporal central con soluciones frías aproximadamente a $4^{\circ} \mathrm{C}$, vasopresor (norepinefrina) con la finalidad de mantener una

\section{Correspondencia}

*Rafael A. Reyes-Monge

E-mail: reyesraphael92@gmail.com
Fecha de recepción: 15-09-2021

Fecha de aceptación: 17-12-2021 DOI: 10.24875/REIE.21000102
Disponible en internet: 07-03-2022

Rev Educ Investig Emer. 2022;4(Supl 1):82-85 www.medicinadeemergencias.com bajo la licencia CC BY-NC-ND (http://creativecommons.org/licenses/by-nc-nd/4.0/). 
Tabla 1. Valores de laboratorio

\begin{tabular}{|c|c|c|}
\hline Ingreso & $6 \mathrm{~h}$ posteriores & 12 h posteriores \\
\hline $\begin{array}{l}\text { Leucocitos } 28,000 \\
\text { Hemoglobina } 14.5 \\
\text { Plaquetas } 5000\end{array}$ & $\begin{array}{l}\text { Leucocitos } 25,330 \\
\text { Hemoglobina } 12.5 \\
\text { Plaquetas } 35,000\end{array}$ & $\begin{array}{l}\text { Leucocitos } 19,800 \\
\text { Hemoglobina } 14.5 \\
\text { Plaquetas } 5000\end{array}$ \\
\hline $\begin{array}{l}\text { Glucosa } 350 \mathrm{mg} / \mathrm{dl} \\
\text { Urea } 25 \mathrm{mg} / \mathrm{dl} \\
\text { Creatinina } 2.3 \mathrm{mg} / \mathrm{dl}\end{array}$ & $\begin{array}{l}\text { Glucosa } 132 \mathrm{mg} / \mathrm{dl} \\
\text { Urea } 68 \mathrm{mg} / \mathrm{dl} \\
\text { Creatinina } 2.6 \mathrm{mg} / \mathrm{dl}\end{array}$ & $\begin{array}{l}\text { Glucosa } 45 \mathrm{mg} / \mathrm{dl} \\
\text { Urea } 117 \mathrm{mg} / \mathrm{dl} \\
\text { Creatinina } 4.1 \mathrm{mg} / \mathrm{dl}\end{array}$ \\
\hline $\begin{array}{l}\text { Albúmina } 4.7 \mathrm{~g} / \mathrm{dl} \\
\text { Fosfatasa alcalina } 135 \mathrm{UI} / \mathrm{l} \\
\text { Bilirrubina total } 3.4 \mathrm{mg} / \mathrm{dl} \\
\text { Bilirrubina directa } 2.3 \mathrm{mg} / \mathrm{dl} \\
\text { Bilirrubina indirecta } 1.1 \mathrm{mg} / \mathrm{dl} \\
\text { TGO } 149 \mathrm{UI} / \mathrm{l} \\
\text { TGP } 180 \mathrm{UI} / \mathrm{l} \\
\text { CPK no hay reactivo }\end{array}$ & & $\begin{array}{l}\text { Albúmina } 2.7 \mathrm{~g} / \mathrm{dl} \\
\text { Fosfatasa alcalina } 287 \mathrm{UI} / \mathrm{l} \\
\text { Bilirrubina total } 4.2 \mathrm{mg} / \mathrm{dl} \\
\text { Bilirrubina directa } 3 \mathrm{mg} / \mathrm{dl} \\
\text { Bilirrubina indirecta } 1.2 \mathrm{mg} / \mathrm{dl} \\
\text { TGO } 320 \mathrm{UI} / \mathrm{l} \\
\text { TGP } 426 \mathrm{UI} / \mathrm{l} \\
\text { CPK no hay reactivo }\end{array}$ \\
\hline $\begin{array}{l}\text { Sodio } 136 \mathrm{mmol} / \mathrm{l} \\
\text { Potasio } 4.4 \mathrm{mmol} / \mathrm{l} \\
\text { Calcio } 10.6 \mathrm{mmol} / \mathrm{l}\end{array}$ & $\begin{array}{l}\text { Sodio } 140 \mathrm{mmol} / \mathrm{l} \\
\text { Potasio } 5.5 \mathrm{mmol} / \mathrm{l} \\
\text { Calcio } 6.7 \mathrm{mmol} / \mathrm{l}\end{array}$ & $\begin{array}{l}\text { Sodio } 136 \mathrm{mmol} / \mathrm{l} \\
\text { Potasio } 6.5 \mathrm{mmol} / \mathrm{l} \\
\text { Calcio } 5.3 \mathrm{mmol} / \mathrm{l}\end{array}$ \\
\hline $\begin{array}{l}\text { Fibrinógeno } 52 \mathrm{mg} / \mathrm{dl} \\
\text { Dímero D } 1.7 \mathrm{mg} / \mathrm{l} \\
\text { Tiempo de protrombina } 19 \mathrm{~s} \\
\text { INR } 2.1 \\
\text { Tiempo de tromboplastina } 50 \mathrm{~s}\end{array}$ & $\begin{array}{l}\text { Fibrinógeno } 153 \mathrm{mg} / \mathrm{dl} \\
\text { Dímero D } 2.4 \mathrm{mg} / \mathrm{l} \\
\text { Tiempo de protrombina } 17 \mathrm{~s} \\
\text { INR } 1.8 \\
\text { Tiempo de tromboplastina } 43 \mathrm{~s}\end{array}$ & \\
\hline \multirow[t]{2}{*}{$\begin{array}{l}\mathrm{pH} 7.30 \\
\mathrm{pCO}_{2} 20 \\
\mathrm{pO}_{2} 80 \\
\mathrm{HCO}_{3} 13 \\
\text { Lactato } 2.7\end{array}$} & $\begin{array}{l}\mathrm{pH} 7.29 \\
\mathrm{pCO}_{2} 43 \\
\mathrm{pO}_{2} 90 \\
\mathrm{HCO}_{3} 9 \\
\text { Lactato } 1.8\end{array}$ & $\begin{array}{l}\mathrm{pH} 7.27 \\
\mathrm{pCO}_{2} 45 \\
\mathrm{pO}_{2} 94 \\
\mathrm{HCO}_{3} 8 \\
\text { Lactato } 2.3\end{array}$ \\
\hline & $\begin{array}{l}\text { Orina: } \\
\text { Densidad } 1.016 \\
\text { Proteínas } 75 \\
\text { pH } 6.34 \\
\text { Hematíes } 30-50 \text { por campo } \\
\text { Leucocitos } 10-20 \text { por campo }\end{array}$ & \\
\hline
\end{tabular}

CPK: creatina fosfocinasa; $\mathrm{HCO}_{3}$ : bicarbonato; INR: International Normalized Ratio; $\mathrm{pCO}_{2}$ : presión parcial de dióxido de carbono; $\mathrm{pO}_{2}$ : presión parcial de oxígeno; TGO: transaminasa glutámico oxalacética; TGP: transaminasa glutámico pirúvica.

presión arterial media (PAM) perfusora, y se realizó reanimación hídrica con soluciones cristaloides encaminada a mantener un adecuado volumen sistólico y una diuresis adecuada; se administraron en total $2500 \mathrm{ml}$ de solución Hartmann, pero el paciente se mantuvo en anuria durante su hospitalización. Para el manejo de la coagulación intravascular diseminada se administraron en total tres plasmas frescos congelados y cinco crioprecipitados, además de una aféresis plaquetaria. Durante la hospitalización (la cual duró aproximadamente 15 horas), pese a su manejo el paciente tuvo una evolución tórpida, con persistencia de la acidosis metabólica, además de continuar en anuria, candidato a la realización de hemodiálisis sin incapacidad de realizarse por motivos no médicos. Persiste la hipotensión con dosis máxima de norepinefrina y finalmente cae en paro cardiorrespiratorio, se otorgan maniobras de reanimación avanzada y no hay retorno a la circulación espontánea.

\section{Discusión}

El golpe de calor es una patología potencialmente mortal que se presenta principalmente en pacientes que no tienen una adecuada adaptación a altas temperaturas y que se desenvuelven en áreas de alta exposición. Dentro del mecanismo de patogénesis se encuentra la transición de una fase termorreguladora compensada (en la cual la pérdida de calor es mayor que la ganancia) a una fase descompensada (caracterizada por un incremento en la ganancia con disminución de la pérdida de calor) ${ }^{3}$. 
De manera inicial, la hipertermia detona una respuesta sistémica de estrés que incluye células endoteliales, leucocitos y células epiteliales, la cual tiene la finalidad de promover la reparación celular. Como consecuencia existe un incremento en las concentraciones plasmáticas de citocinas proinflamatorias. Ante una prolongación en la exposición a las altas temperaturas se incrementa la demanda metabólica, desarrollando efectos citotóxicos que condicionan una desregulación de la reacción inflamatoria. El estado hipermetabólico lleva a un incremento en el gasto cardiaco, ocasionando un descenso en el flujo sanguíneo visceral. Existe, además, un incremento en la permeabilidad gastrointestinal que causa diarrea. Se produce colapso cardiovascular y, a consecuencia de este proceso, una disminución en el flujo sanguíneo renal que explica la lesión renal aguda y la acidosis metabólica. Ante la respuesta inflamatoria sistémica desregulada existen alteraciones en la cascada de la coagulación, la cual finalmente va a devenir en coagulación intravascular diseminada ${ }^{4}$.

Dentro del abordaje en el área de urgencias deben realizarse paraclínicos incluyendo biometría hemática, química sanguínea, gasometría arterial, creatina fosfocinasa, electrolitos séricos, examen general de orina y pruebas de funcionamiento hepático ${ }^{3}$.

Para establecer el diagnóstico no es necesaria la toma de paraclínicos, pues se basa en la tríada de hipertermia $\left(>40.3^{\circ} \mathrm{C}\right)$, alteraciones neurológicas y exposición reciente a altas temperaturas ${ }^{4}$.

Se debe iniciar reanimación hídrica con soluciones isotónicas, ya que la deshidratación no es un problema primario. Ante la elevación de la temperatura corporal central hay que buscar disminuirla con soluciones frías $\left(4{ }^{\circ} \mathrm{C}, 1000 \mathrm{ml} / 30 \mathrm{~min}\right.$ ) hasta ser $<38^{\circ} \mathrm{C}^{5}$. Para el manejo de las crisis convulsivas se deben administrar benzodiacepinas de manera repetida 0 inclusive fenitoína (dosis de carga $15-20 \mathrm{mg} / \mathrm{kg}$ en $15 \mathrm{~min}$ ) hasta el cese de las convulsiones ${ }^{6}$. Para el manejo de la falla cardiaca se puede iniciar dobutamina $V(1 \mu \mathrm{g} / \mathrm{kg} / \mathrm{min}$ y posteriormente $2-20 \mu \mathrm{g} / \mathrm{kg} / \mathrm{min})^{7}$. En el manejo de la lesión renal aguda se busca mantener un volumen urinario $>50 \mathrm{ml} / \mathrm{kg} / \mathrm{h}^{8}$. En caso de deterioro neurológico $<9$ se debe asegurar la vía aérea.

Dentro de las complicaciones se incluye la rabdomiólisis, y en este caso se debe mantener una infusión intravenosa de 1-2 $\mathrm{l} / \mathrm{h}$ con la finalidad de mantener unos volúmenes urinarios de $300 \mathrm{ml} / \mathrm{h}^{8}$. Cuando existe coagulación intravascular diseminada, en caso de sangrado, el tratamiento va encaminado a administrar plasma fresco congelado en dosis de bolo de $10-15 \mathrm{ml} / \mathrm{kg}$ y luego $200-400 \mathrm{ml}$, y $5-10$ unidades de crioprecipitados por transfusión. Si el fibrinógeno es < $180 \mathrm{mg} / \mathrm{dl}$ se deben administran concentrados plaquetarios $^{9}$. Si existe falla hepática, se puede administrar $\mathrm{N}$-acetilcisteína en bolo de $150 \mathrm{mg} / \mathrm{kg}$ en infusión para 20 minutos, luego $50 \mathrm{mg} / \mathrm{kg}$ para 4 horas y finalmente $100 \mathrm{Mg} / \mathrm{Kg}$ para 16 horas $^{10}$.

En el caso particular de este paciente, se realizó el manejo de acuerdo con las recomendaciones internacionales más adecuadas, administrando crioprecipitados, plasma y aféresis plaquetaria. Durante su estancia en el hospital continuó con anuria, además de persistir con acidosis, y claramente era candidato a terapia de sustitución renal, la cual no pudo realizarse. Se encontraba con vasopresor a dosis máxima, con indicación de administrar un segundo vasopresor. Para la falla hepática se reporta el uso de $\mathrm{N}$-acetilcisteína, la cual no pudo ser administrada. No se pudo determinar una posible rabdomiólisis al no contar con reactivo para la creatina fosfocinasa; ante esta posibilidad diagnóstica, se hubiese optado por otras medidas terapéuticas.

\section{Conclusiones}

El golpe de calor es una condición potencialmente fatal, infradiagnosticada y con elevada morbimortalidad si el diagnóstico y el tratamiento no son tempranos. Se asocia a menudo con disfunción orgánica que de manera rápida conduce a la muerte. El pilar del tratamiento es la disminución de la temperatura central, ya que la supervivencia se ve relacionada con la velocidad de descenso de esta. El manejo de sostén es de la falla orgánica múltiple.

\section{Agradecimientos}

Agradecemos al personal que labora en el Hospital General del Estado de Sonora, porque gracias al trabajo en equipo podemos obtener los mejores resultados para nuestros pacientes.

\section{Financiamiento}

El presente trabajo no ha recibido ninguna beca específica de agencias de los sectores públicos 0 privados. 


\section{Conflicto de intereses}

Los autores declaran no tener conflictos de intereses.

\section{Responsabilidades éticas}

Protección de personas y animales. Los autores declaran que para esta investigación no se han realizado experimentos en seres humanos ni en animales.

Confidencialidad de los datos. Los autores declaran que en este artículo no aparecen datos de pacientes.

Derecho a la privacidad y consentimiento informado. Los autores declaran que en este artículo no aparecen datos de pacientes.

\section{Bibliografía}

1. Epstein Y, Yanovich R. Heatstroke. N Engl J Med. 2019;380:2449-59.

2. Leon LR, Bouchama A. Heat stroke. Compr Physiol. 2015;5:611-47.

3. Hifumi T, Kondo Y, Shimizu K, Miyake Y. Heat stroke. J Intensive Care. 2018:6:30.

4. Morris A, Patel G. Heat stroke. StatPearls. 2021 Jun 25. Treasure Island (FL): StatPearls Publishing; 2021

5. Gaudio FG, Grissom CK. Cooling methods in heat stroke. J Emerg Med. 2016;50:607-16

6. Cheshire WP Jr. Thermoregulatory disorders and illness related to heat and cold stress. Auton Neurosci. 2016;196:91-104.

7. Cheshire WP Jr. Thermoregulatory disorders and illness related to heat and cold stress. Auton Neurosci. 2016;196:91-104.

8. Stanley M, Chippa V, Aeddula NR, Quintanilla Rodríguez BS, Adigun R. Rhabdomyolysis. StatPearls. 2021 Jul 10. Treasure Island (FL): StatPearIs Publishing; 2021.

9. Costello RA, Nehring SM. Disseminated intravascular coagulation. StatPearls. 2021 Jul 17. Treasure Island (FL): StatPearls Publishing; 2021.

10. Woolbright BL, Jaeschke $H$. Role of the inflammasome in acetaminophen-induced liver injury and acute liver failure. J Hepatol 2017;66:836-48 